\title{
Optimal Spacing Design for Pilots in OFDM Systems over Multipath Fading Channels
}

\author{
Youssefi My Abdelkader, El Abbadi Jamal \\ Laboratoire d'Electronique et Communication, EMI-Rabat, Morocco \\ E-mail: \{ab.youssefi,j.elabbadi\}@gmail.com \\ Received July 21, 2010; revised August 31, 2010; accepted September 29, 2010
}

\begin{abstract}
In wireless orthogonal frequency division multiplexing (OFDM) systems, the time-varying channel is often estimated by algorithms based on pilot symbols. Such an estimator, however, requires statistical prior knowledge that is not easily obtained. Therefore, the pilot tones have to be close enough to fulfill the sampling theorem. In this case the statistical knowledge of the channel is not required to reconstruct correctly the channel impulse response (CIR). This paper explores the optimal placement and number of pilot symbols, we investigate optimal training sequences in OFDM systems and we analyze the number of pilot symbols required to fulfill the sampling theorem. Using a general model for a multipath slowly fading channel, the approach is based on the LS as a criterion of channel estimation while the channel interpolation is done using the piecewise-constant interpolation compromising between complexity and performance. Simulation results demonstrate the good performance of our approach.
\end{abstract}

Keywords: OFDM, Multipath Channel, Channel Estimation, Pilot Symbols

\section{Introduction}

OFDM (Orthogonal Frequency Division Multiplexing) has been widely applied in wireless communication systems due to its high data rate transmission and its robustness to multipath channel delay [1,2]. The channel characteristics must be known at the receiver to recover the transmitted signal after demodulation, so channel estimation is needed.

Two classes of methods are available for the receiver to acquire channel state information (CSI): One is based on training symbols that are a priori known to the receiver, whereas the other relies only on the received symbols to acquire CSI blindly.

Relative to training, blind schemes typically require longer data records and entail higher complexity.

In OFDM systems, channel estimation is usually performed by sending training pilot symbols on sub-carriers known at the receiver. The quality of the estimation depends on the pilot arrangement. The channel estimation can be performed by either inserting pilot tones into all of the subcarriers of OFDM symbols with a specific period (Block-type) or inserting pilot tones into each OFDM symbol (Comb-type) [3]:

1) Block-type Arrangement (shows in Figure1a):
The block type pilot channel estimation, has been developed under the assumption of slow fading channel, this assumes that the channel transfer function is not changing very rapidly. In block-type pilot based channel estimation, OFDM channel estimation symbols are transmitted periodically, in which all sub-carriers are used as pilots. If the channel is constant during the block, there will be no channel estimation error since the pilots are sent at all carriers. The estimation can be performed by using either LS or MMSE.

2) Comb-type Arrangement (shows in Figure1b): the comb-type pilot channel estimation has been introduced to satisfy the need for equalizing when the channel changes even in one OFDM block. The comb-type pilot channel estimation consists of algorithms to estimate the channel at pilot frequencies and to interpolate the channel.

This work is based on a Comb-type arrangement, Comb-type pilot pattern is more appropriate for the estimation of time varying channels [4]. The channel is considered to be frequency-selective invariant over each received block, but is allowed to vary from block-toblock.

The design of a channel estimator is based on two fundamental problems: 


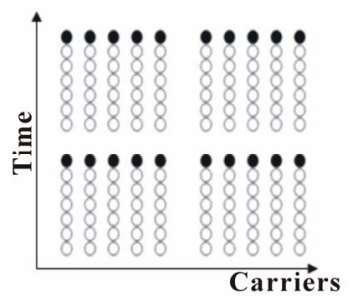

(a)

Block-type pilot arrangement

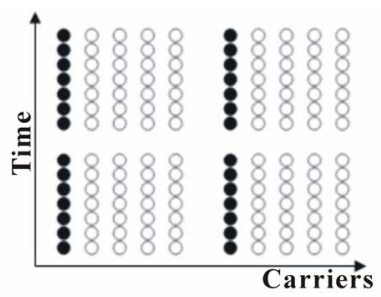

(b)

Comb-type pilot arrangement

Figure 1. Pilot arrangement

- The amount of pilot symbols to be transmitted.

- The complexity of the estimator.

The MMSE estimator has good performance but high complexity. The LS estimator has low complexity, but its performance is not as good as that of the MMSE estimator [5]. This work is based on the LS as a criterion of channel estimation because LS and MMSE criteria exhibit similar performance in high SNR regimes. The OFDM transmission technique is robust to multi-path channel delay, cyclic prefix extension is used to eliminate inter-symbol interference (ISI), but the Inter-carrier interference (ICI) remains a problem because of Doppler frequency. In fast fading channels when the channel changes even in one OFDM block, orthogonality of the tones can't be preserved because of Doppler Effect [6]. Studying the optimum spacing of the pilots in the general case shows that the optimum time-frequency spacing depends upon the channel parameters (the Doppler frequency and the power delay of the channel) [7]. Therefore, the OFDM technique is extremely attractive for slowly fading multipath channel as that usually used in WLAN systems or in indoor environment.

In this paper we study the case of multipath slowly fading channels while the orthogonality of tones is preserved. The optimal spacing of pilot symbols for OFDM systems has been investigated by several studies over the past ten years. In literature, the results of work based on channel capacity investigating this optimization problem [8] confirm the results of the study based on the OFDM linear system $(\mathrm{Y}=\mathrm{HX}+\mathrm{N})$, these studies show that in a multipath channel with $\mathrm{L}$ independent paths channel es- timation requires at least L pilot symbols equally- spaced in the OFDM block.

In this work we explore the optimization of pilot subcarriers required for channel estimation and optimal positions of pilots. We show that the application of sampling theorem confirms the result of previous works.

In Section 2, we describe the channel estimation based on a Comb-type arrangement. Section 3 discusses the optimal placement and number of pilot symbols in an OFDM frame. Simulation results are presented in Section 4.

\section{OFDM Channel Estimation Based on Comb-Type Pilot Arrangement}

\subsection{Notations}

Standard notations are used in this paper.

$\begin{array}{ll}(.)^{t} & \text { Transpose } \\ (.)^{*} & \text { Hermitian } \\ (.) & \text { Estimate of }(.) \\ (.)^{(p l)} & \text { Vector with pilot tone subscripts } \\ E\{.\} & \text { Expectation operator } \\ \operatorname{tr}\{.\} & \text { Trace operator } \\ |\cdot| & \text { Absolute value } \\ \|\cdot\| & \text { 2-norm } \\ I_{n} & \text { n x n Identity matrix } \\ N & \text { Number of OFDM tones } \\ T & \quad \text { The sampling interval } \\ T & \text { The OFDM block duration }(T=N . T S) \\ L & \text { Maximum length of channel } \\ K & \text { Frequency subscript (tone number) } \\ m & \text { Time subscript (OFDM symbol number) } \\ Y_{k, m} & \text { Received signal } \\ X_{k, m} & \text { Transmitted data } \\ h_{m} & \text { Channel impulse response } \\ H_{m} & \text { Channel matrix } \\ N_{k, m} & \text { AWGN noise } \\ F_{d} & \text { Doppler frequency } \\ W_{N}=e^{-j 2 \pi / N} & \end{array}$

\subsection{Channel Estimation Based on Comb-Type Pilot Arrangement}

In an OFDM system, the received signal at the $\mathrm{k}^{\text {th }}$ tone can be expressed as

$$
Y(k)=H(k) X(k)+I(k)+N(k), k=0,1, \ldots N-1
$$

where $I(k)$ the inter-carrier interference (ICI) because of Doppler frequency. 
In the case of multipath slowly fading channels, when the channel is frequency-selective invariant over each received block OFDM symbol, the orthogonality between subcarriers can be fully preserved, and we can write

$$
Y_{k, m}=H_{k, m} X_{k, m}+N_{k, m}
$$

There are several criteria used for channel estimation, for reasons of complexity, the estimation can be performed by using either linear MMSE criterion (Wiener filtering) $[9,10]$ and LS criterion. However, in high SNR regimes and when the noise level is low, LS criterion offers a good compromising performance / complexity.

1) The estimate of the channel at pilot sub-carriers based on LS estimation is given as follows,

Assume that each OFDM symbol has $N$ subcarriers where pilots occupy p subcarriers. By LS estimation, the channel state information (CSI) at pilot tones in the $\mathrm{k}^{\text {th }}$ OFDM symbol can be obtained as

$$
\hat{H}_{k, m}=\frac{Y_{k, m}}{X_{k, m}}
$$

where $k$ is the pilot tones, $k=\left\{k_{1}, k_{2} \ldots, k_{p}\right\}$

\section{2) Interpolation}

In comb-type pilot based channel estimation, an interpolation technique is necessary in order to estimate channel at data sub-carriers by using the channel information at pilot sub-carriers [11]. The channel interpolation is done using the piecewise-constant interpolation, linear interpolation, second order interpolation, low-pass interpolation, spline cubic interpolation...In the following Figure 2, there's a comparison between three types of frequency channel response, Indoor A, Pedestrian B and Vehicular B [12]:

The pilot symbols have to be close enough to fulfill the sampling theorem and avoid aliasing. Therefore, with the piecewise-constant interpolation we can reconstruct correctly the channel impulse response as it will be shown in this paper. Whereas, when we have enough information about channel characteristics, we can choose a type of interpolation to estimate the channel (even if

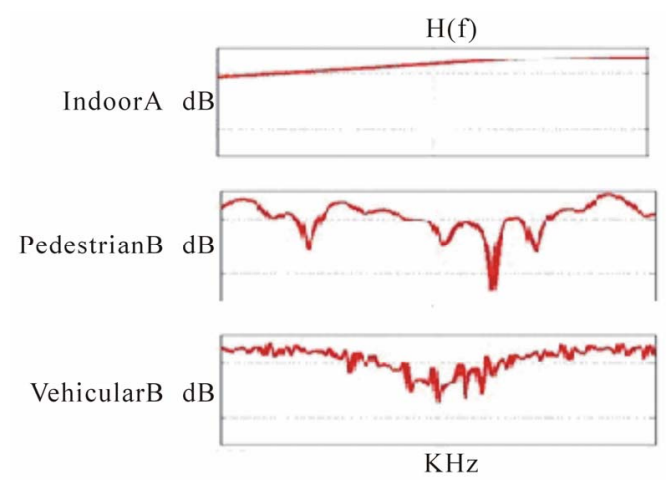

Figure 2. Rayleigh model 2GHz, ETSI TR101-102. pilot symbols aren't close enough to fulfill the sampling theorem).

The channel estimation $H_{e}(k)$ at the data-carrier $k(=0,1, \ldots, N-1)$ is given as follows,

a) $H_{e}(k)$ at pilot tones is used to find the estimated transmitted signal $X_{e}(k)$, we can get

$$
X_{e}(k)=\frac{Y(k)}{H_{e}(k)}, k=\left\{k_{1}, k_{2} \ldots, k_{p}\right\}
$$

Figure $3 X_{e}(k)$ is mapped to the constellation and then obtained $X_{\text {pure }}(k)$.

b) The estimated channel $X_{e}(k)$ at pilot tones is updated:

$$
H_{e}(k)=\frac{Y(k)}{X_{\text {pure }}(k)}, k=0,1 \ldots, N-1
$$

The channel estimation $H_{e}(k)$ at the data-carrier $k$ $(k=0.1, \ldots, N-1)$ is given using an efficient interpolation technique in order to estimate channel at data sub-carriers by using the channel information at pilot sub-carriers.

\section{The Minimum Number of Pilot Tones}

In this section, we present some answers to the question of minimum number of pilots needed for channel estimation in OFDM systems. This problem has been the subject of several investigations, the theoretical study shows that the channel estimation requires at least $\mathrm{L}$ pilot symbols, and we can prove this result by two different methods. One is based on the sampling theorem, whereas the other relies only on the study of the OFDM linear system $Y=H X+N$.

\subsection{The Application of Shannon's Sampling Theorem}

The channel estimation with a Comb-type pilot arrangement is based on an insertion of constant values called pilots in some sub-carriers of the OFDM frame. Figure 4.

After the signal demodulation, an LS estimator gives

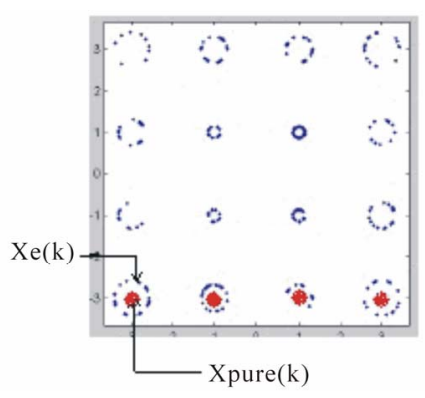

Figure 3. Constellation. 


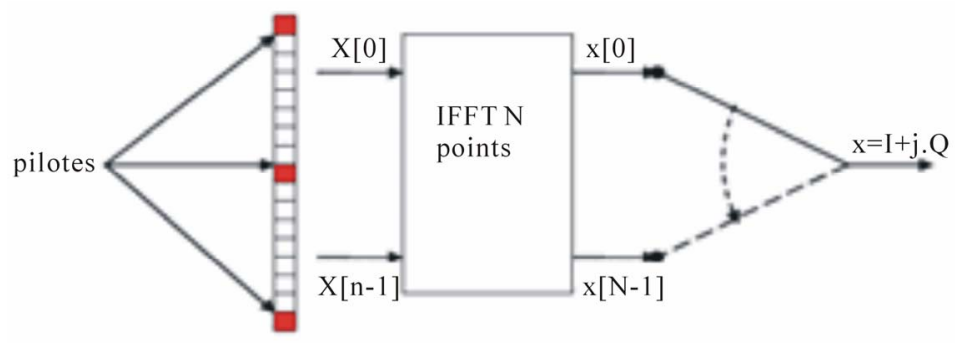

Figure 4. Pilot insertion.

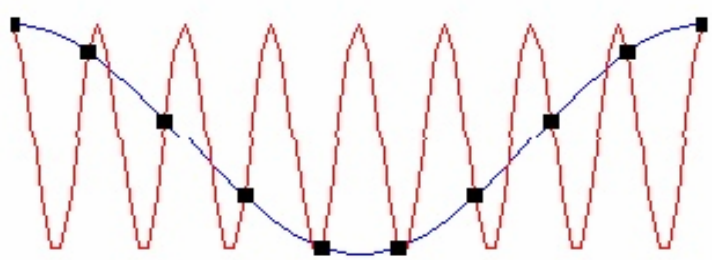

Figure 5. Sampling process.

channel estimation at pilot sub-carriers. The piecewiseconstant interpolation is used in order to estimate channel at the values between the pilot samples as it's shown in Figure 5.

Therefore, the process of channel estimation based on pilot symbols with piecewise constant interpolation is similar to the sampling process. The BER can be lowered by placing the pilot symbols closer than that specified by the sampling theorem.

\section{- $\quad$ Multipath channel modeling}

The complexity of an estimator depends on the number of the propagation paths (determined by the ratio between the maximum delay spread $\tau_{\text {max }}$ and the sampling period $T s$ ). For example, in BRAN A channel there's 8 paths (the propagation channel for an office environment as part of the standard HiperLAN2-5Ghz). In the analogue domain, the multipath channel is described by the following complex-valued impulse response [13]:

$$
h(t, \tau)=\sum_{l=1}^{L} \alpha_{l}(t) \delta\left(\tau-\tau_{l}(t)\right)
$$

where $\alpha_{1}$ is the complex gain of the lth path, $\tau_{1}$ is the propagation delay for the $l$ th path. In digital transmission systems, symbols are often transmitted in regular time interval Ts, called the sampling period. This period is often small in comparison with the maximum delay time $\tau_{\text {max }}$.

The discrete multipath model is regarded as a reference for most of the contemporary wideband terrestrial wireless systems under a reasonable approximation of constant number of multipath components $L$ and slow variation of the delay values $\tau_{1}(\mathrm{t})$. The multipath channel is then modeled as

$$
h(t, \tau)=\sum_{l=1}^{L} \alpha_{l}(t) \delta\left(\tau-\tau_{l} \cdot T_{s}\right)
$$

The path gains are given as samples taken at different period $T s$ (see Figure 5).

\section{- $\quad$ The application of sampling theorem}

The spectrum of a discrete signal is a periodic signal (see Figure 6). We will explore the duality of the Fourier transform then we can apply the sampling theorem on the frequency response of the channel.

Since the inter-carrier spacing is $\Delta f$ and the duration of an OFDM symbol is $T=N . T s$, we have $\Delta f=\frac{1}{T}=\frac{1}{N T_{s}}$

If we assume that pilots are placed $N_{f}$ subcarriers apart in every OFDM symbols (Comb-type pilot arrangement), and the pilot symbols are equally-spaced and the spacing
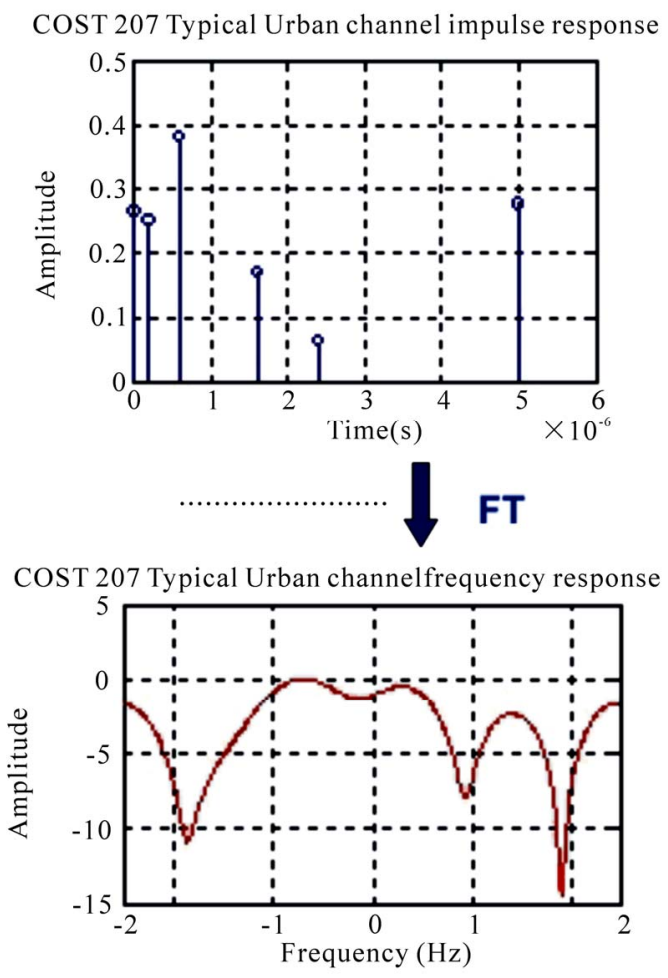

Figure 6. Typical multipath channel response. 
between two consecutive pilot symbols is equal to $f_{e}$, we have

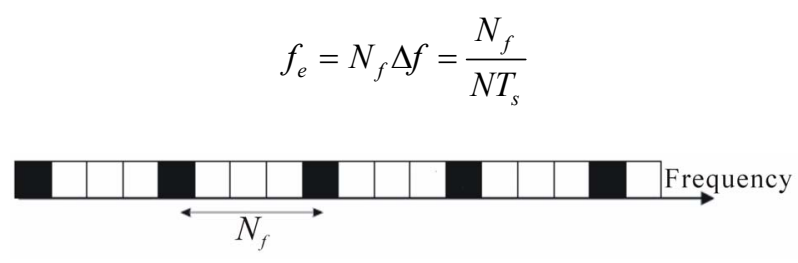

Figure 7. Structure of pilot symbols for $N_{f}=4$

Let $H_{e}(f)$ the frequency response of the channel constructed from the pilot symbols with a piecewise constant-interpolation.

$$
\begin{aligned}
& H_{e}(f)=\sum_{n=-\infty}^{+\infty} H\left(n f_{e}\right) \delta\left(f-n f_{e}\right) \\
& =H(f) \sum_{n=-\infty}^{+\infty} \delta\left(f-n f_{e}\right)
\end{aligned}
$$

The inverse Fourier transform of this distribution is $h_{e}$ $(t)$ :

$$
\begin{aligned}
& h_{e}(t)=h(t) * T F^{-1}\left(\sum_{n=-\infty}^{+\infty} \delta(f-n f)_{e}\right) \\
& =h(t) * \frac{1}{f_{e}} \sum_{n=-\infty}^{+\infty} \delta\left(t-\frac{n}{f_{e}}\right) \\
& =\frac{1}{f_{e}} \sum_{n=-\infty}^{+\infty} h\left(t-\frac{n}{f_{e}}\right) \\
& =T_{e} \sum_{n=-\infty}^{+\infty} h\left(t-n T_{e}\right)
\end{aligned}
$$

The inverse Fourier transform of this distribution is $h_{e}$ $(t)$ :

$$
\begin{aligned}
& h_{e}(t)=h(t) * T F^{-1}\left(\sum_{n=-\infty}^{+\infty} \delta\left(f-n f_{e}\right)\right) \\
& =h(t) * \frac{1}{f_{e}} \sum_{n=-\infty}^{+\infty} \delta\left(t-\frac{n}{f_{e}}\right) \\
& =\frac{1}{f_{e}} \sum_{n=-\infty}^{+\infty} h\left(t-\frac{n}{f_{e}}\right) \\
& =T_{e} \sum_{n=-\infty}^{+\infty} h\left(t-n T_{e}\right)
\end{aligned}
$$

Then we can reconstruct $h(t)$ from $h_{e}(t)$. We need a time-domain windowing to reconstruct the time-domain channel impulse response (CIR).

The samples have to be close enough to fulfill the sampling theorem and avoid aliasing. So, we must choose the sampling period $T e$ greater than the channel impulse response length.

Let $h_{m}=\left[h_{0, m}, h_{1, m}, \ldots, h_{L-1, m}\right]^{t}$ the channel impulse re- sponse.

The length of the multipath channel impulse response is $L . T s$. To avoid aliasing, we must respect the following inequality $T_{e} \geq L . T_{s}$.

Knowing that $f_{e}=N_{f} \Delta f=\frac{N_{f}}{N T_{s}}$

we have $T_{e}=\frac{N . T_{s}}{N_{f}}$

however, we have the following inequality

$$
\frac{N . T_{s}}{N_{f}} \geq L . T_{s} \quad \rightarrow \quad \frac{N}{N_{f}} \geq L
$$

We will show later that the equispaced pilot symbols are optimal in the sense of MMSE.

Because equispaced placement of pilot symbols is impossible when $N / N_{f}$ is not an integer, we will consider only the case when $N / N_{f}$ is an integer.

For this special case we have

$$
\text { Number_of_Pilot_Tones }=\frac{N}{N_{f}} \geq L
$$

knowing that $\frac{N}{N_{f}}$ is the number of pilot symbols in the OFDM frame, then the channel estimation requires at least $L$ pilot symbols.

\subsection{OFDM Linear System $\mathbf{Y}=\mathbf{H X}+\mathbf{N}$}

In the OFDM system when orthogonality is preserved, the system equations can be expressed as

$$
Y_{k, m}=H_{k, m} X_{k, m}+N_{k, m}
$$

where $N_{k, m}$ AWGN noise.

Let $\left\{k_{1}, k_{2}, \ldots, k_{L}\right\}$ be the set of $L$ tones used for transmitting pilot symbols.

In the absence of noise, from (1) we can write:

$$
Y^{p l}=H^{p l} X^{p l}
$$

where

$$
H^{p l}=\frac{1}{\sqrt{N}} \underbrace{\left(\begin{array}{cccc}
1 & W_{N}^{k_{1}} & \ldots & W_{N}^{k_{1}(L-1)} \\
1 & W_{N}^{k_{2}} & \ldots & W_{N}^{k_{2}(L-1)} \\
\ldots & \ldots & \ldots & \ldots \\
1 & W_{N}^{k_{L}} & \ldots & W_{N}^{k_{L}(L-1)}
\end{array}\right)}_{Q} h
$$

Since $Q$ is a Vandermonde matrix with $L$ parameters distinct, $h$ can be found exactly by inverting $Q$.

With the system equations (1), we have $L$ unknowns $h_{1}, \ldots h_{L}$

- If the number of pilot symbols is equal to $L$, then the 
number of equations equals the number of unknowns, then the system can be solved.

- If the number of pilot symbols is less than $L$, the number of equations is less than the number of unknowns then we have an under-determined system of linear equations [14], and hence a non-unique solution $h$.

Therefore, in both cases (absence or presence of noise), $L$ pilot symbols are the minimum number required for exact channel estimation.

\subsection{Optimum Set of Pilot Tones}

It has been found in $[14,15]$ that in the presence of AWGN noise, the MMSE estimate of $h$ occurs when the pilot symbols are equally-spaced in the OFDM frame.

Proof:

From (1) we can write:

$$
R_{k, m}=\frac{Y_{k, m}}{X_{k, m}}=H_{k, m}+\frac{S_{k, m}}{\left|X_{k, m}\right|}, k=0,1, \ldots, N-1
$$

where $S_{k, m}=\frac{N_{k, m}}{e^{j \arg X_{k, m}}}$

from (3) we have

$$
H^{p l}=Q \cdot h
$$

from $(4,5)$ the MMSE estimate of $h$ is given by:

$$
\widehat{h}=Q^{(p l)-1} R^{(p l)}
$$

Knowing that each pilot $X_{k, m}$ carries data of constant modulus $\left|X_{k, m}\right|=\sqrt{\varepsilon_{x}}$, from (4) and (6) we have:

$$
\begin{gathered}
\hat{h}=h+Q^{(p l)-1} \frac{1}{\sqrt{\varepsilon_{x}}} S^{(p l)} \\
R^{(p l)}=\left[R_{k_{1}}, \ldots, R_{k_{L}}\right]^{t}, S^{(p l)}=\left[S_{k_{1}}, \ldots, S_{k_{L}}\right]^{t}
\end{gathered}
$$

The matrix $Q^{(p l)}$ depends on the choice of the set of pilot tones. Thus, the design problem is to choose the set that minimizes the MSE $E\left\{\|\hat{h}-h\|^{2}\right\}$. This can be obtained as follows,

$$
\begin{gathered}
E\left\{\|\hat{h}-h\|^{2}\right\}=\frac{1}{\varepsilon_{x}} \operatorname{tr}\left\{Q^{(p l)-1} \sigma^{2}{ }_{s} I_{L} Q^{(p l)-I^{*}}\right\} \\
=\frac{\sigma_{s}^{2}}{\varepsilon_{x}} \operatorname{tr}\left\{\left(Q^{(p l)^{*}} Q^{(p l)}\right)^{-1}\right\} \\
Q^{(p l)^{*}} Q^{(p l)} \text { can be expressed as }
\end{gathered}
$$

where $\operatorname{tr}\left(Q^{(p l)^{*}} Q^{(p l)}\right)=\frac{L^{2}}{N}$

Let $\lambda_{1}, \lambda_{2}, \ldots, \lambda_{L}$ the eigenvalues of $\left(Q^{(p l)^{*}} Q^{(p l)}\right)^{-1}$, then the eigenvalues of $\left(Q^{(p l)^{*}} Q^{(p l)}\right)^{-1}$ are $1 / \lambda_{1}, 1 / \lambda_{2}, \ldots, 1 /$ $\lambda_{L}$

Since the trace of a matrix is the sum of its eigenvalues, hence, the relaxed optimization problem is

$$
\begin{gathered}
\operatorname{minimize} \sum_{p=1}^{L} \lambda_{p} \\
\text { Where } \sum_{p=1}^{L} \frac{1}{\lambda_{p}}=\frac{L^{2}}{N} .
\end{gathered}
$$

Since the matrix $\left(Q^{(p l)^{*}} Q^{(p l)}\right)^{-1}$ is non-negativedefinite, all the eigenvalues are non-negative. So, the minimization occurs if all the eigenvalues $\lambda i(i=1, . ., L)$ are identical. This occurs if:

$$
Q^{(p l)^{*}} Q^{(p l)}=\frac{L}{N} I_{L}
$$

Therefore, the optimal sets of tones are

$$
\left\{i, i+\frac{N}{L}, \ldots, i+\frac{(L-1) N}{L}\right\}
$$

where $i=0,1, \ldots, \frac{N}{L}-1$.

\section{Simulation Results}

\subsection{Effect of Multipath Channel}

To validate our analysis, we test an OFDM 16 QAM transmission over a multipath channel in the absence of noise (4 paths, 6 paths and 10 paths) with $\mathrm{N}=256$ subcarriers, and cyclic prefix length equal to $\mathrm{CP}=32$.

In all the following simulations we have chosen the cyclic prefix to be greater than the maximum delay spread in order to avoid inter-symbol interference (shows in Figure 7).

In Figure. 9 we can see clearly the effect of multipath channel; we notice that as the multipath channel length increases, the error rate increases. Channel estimation is necessary to correct the OFDM symbols.

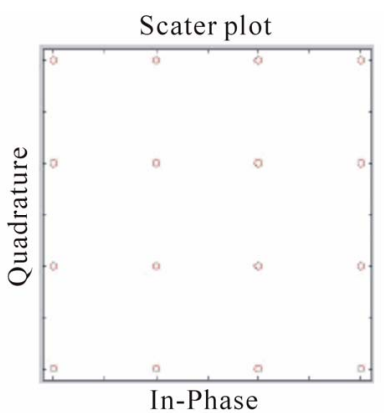

Figure 8. 16-QAM constellation. 


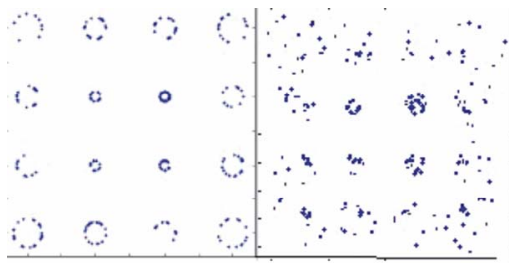

$\mathrm{L}=4$ paths
$\mathrm{L}=6$ paths

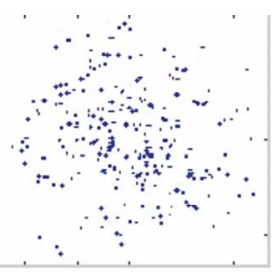

$\mathrm{L}=10$ paths
Figure 9. Effect of multipath channel.

\subsection{Channel Estimation Based on Pilot Symbols}

We consider a typical OFDM system, where $N=256$ subcarriers, and cyclic prefix length equal to $\mathrm{CP}=32$ and 16 QAM transmission over a multipath channel in the absence of noise (8 paths).

We also insert equally-spaced pilot symbols $(1+j)$, the receiver uses an LS estimator and interpolation. We deal with two cases:

1) We insert 16 pilot symbols in every OFDM frame, then the number of pilot symbols is greater than the channel length ( $L=8$ paths).

2) We insert 8 pilot symbols in every OFDM frame, then the number of pilots is equal to the channel length ( $L=8$ paths).

The simulation results are shown in Figure $\mathbf{1 0}$ and Figure 11.

We notice that the channel estimation with 16 pilot symbols /OFDM frame have good performance as it is shown in the Figure11. Otherwise, using 8 pilots/OFDM

\subsection{Optimal Spacing of Pilot Symbols}

We consider a typical OFDM system, where $\mathrm{N}=256$ subcarriers, and cyclic prefix length equal to $\mathrm{CP}=32$ and 64 QAM transmission over a multipath channel. We also

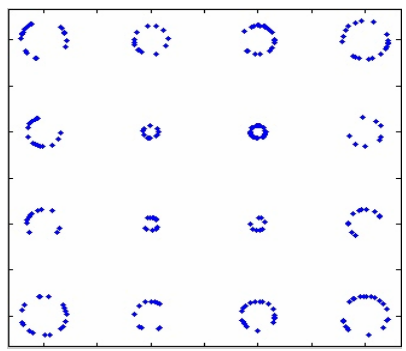

Figure 10. Signal constellation before correction.

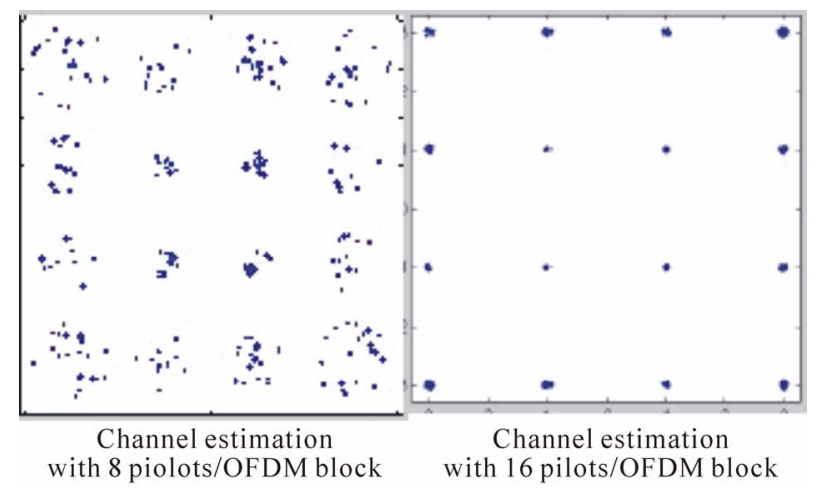

Figure 11. Constellation after correction.

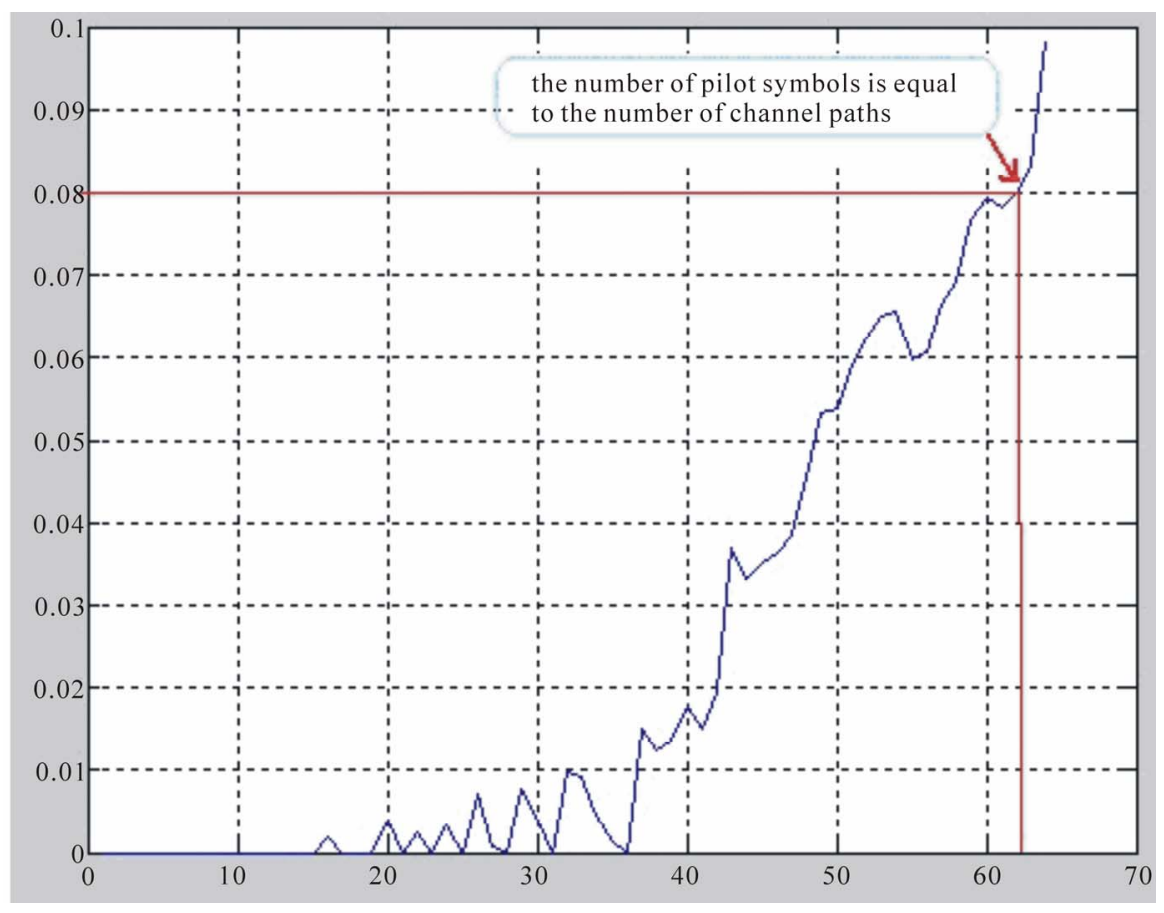

Figure 12. BER versus pilot spacing (4 paths). 


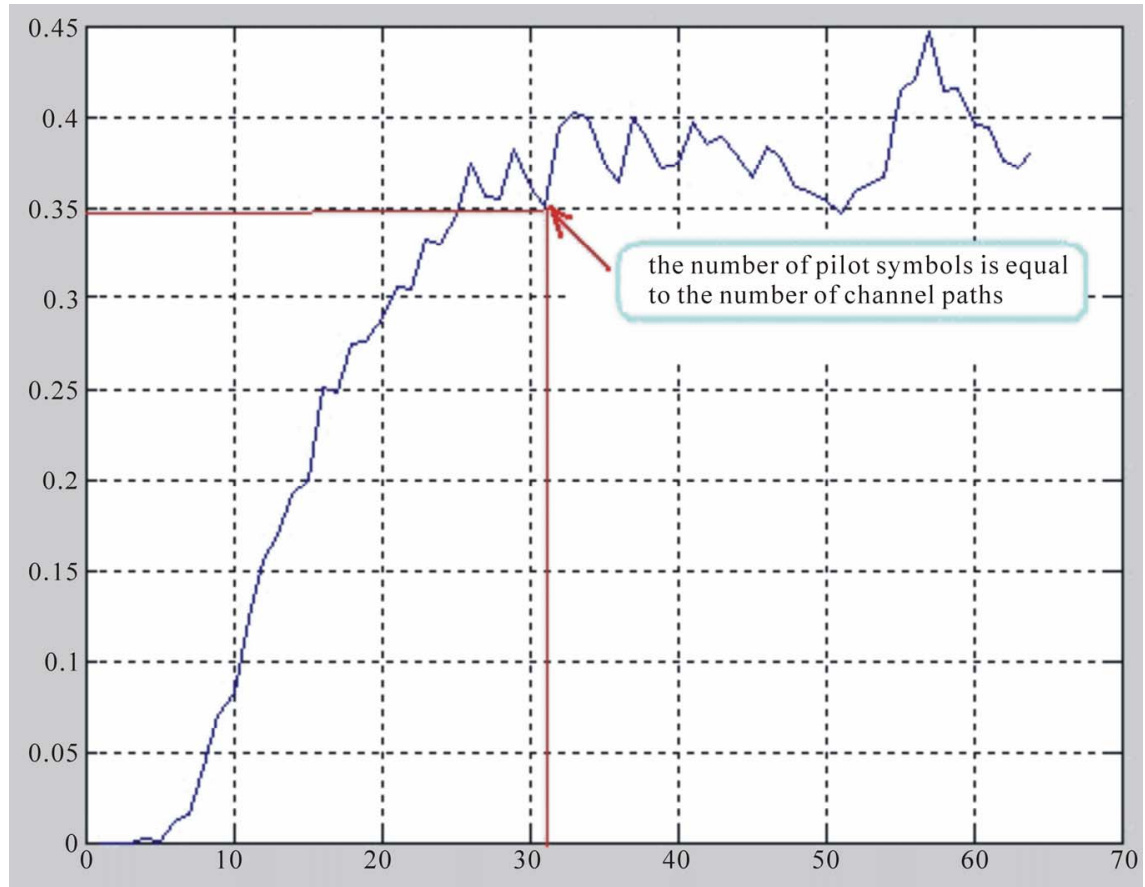

Figure 13. BER versus pilot spacing (8 paths).

frame, this number is not enough for the channel estimation (we have to choose the number of pilot symbols greater than the multipath channel length). insert pilot symbols $(1+j)$, the pilot symbols are not always equally-spaced in the OFDM frame. We vary the number of pilot symbols $(1+j)$ in OFDM blocks. The receiver uses an LS estimator and interpolation.

We deal with two cases:

1) The multipath channel have 4 independents paths (impulse response $\left.=\left[\begin{array}{llll}1 & 0.1 & 0.2 & 0.1\end{array}\right]\right)$, Figure 12 shows the bit error rate (BER) versus pilot spacing in the frequency grid $N_{f}$.

We notice in Figure 12 that when we increase the number of pilot symbols (decreasing $N_{f}$ ) the BER decreases and becomes almost zero at one level of $N_{f}$. When the number of pilot symbols is equal to the number of independent channel paths (pilot spacing $N_{f}=62$, number of pilots/OFDM frame $=4$ ) the BER is about 0.08 , which is acceptable. When the number of pilot symbols is less than $4\left(N_{f}>62\right)$ we can see fast BER increase, which means that the quantity of pilot symbols is not enough to estimate the channel.

2) The multipath channel have 8 independent paths (impulse response $=\left[\begin{array}{llllllll}1 & 0.1 & 0.5 & 0.2 & 0.1 & 1 & 0.2 & 1\end{array}\right]$ ), Figure 13 shows the bit error rate (BER) versus pilot spacing in the frequency grid $N_{f}$.

Theoretical results show that the equally-spaced pilot symbols are optimal in the sense of MMSE, and this optimal placement of pilot symbols is impossible when $N$ /
$N_{f}$ is not an integer. In Figure 12 and Figure 13 we can see clearly that when pilot symbols are equally-spaced in the OFDM frame ( $N / N_{f}$ is an integer), the curve has a local minimum $\left(N_{f}=16,32,64\right)$. Otherwise, the bit error rate takes the smallest value in a given neighborhood.

\section{Conclusions}

In this paper, we have studied the optimum spacing of OFDM pilot symbols in the multipath slowly fading channel. It was shown that channel estimation requires at least L pilot symbols equally-spaced. We proved that the sampling theorem gives the minimum number of pilot tones and confirm the results of previous investigations, and with simulations, we validated the theoretical results.

To approach the theoretical results of the estimation problem, we have to reduce the noise effect on received pilot symbols with high SNR regimes. We have treated the case of invariant multipath channel over each received OFDM block. But this work offers the opportunity to study the optimization problem in fast fading channels with high mobility, which remains an open research area.

\section{Acknowledgements}

The authors would like to thank all the anonymous reviewers of the paper. The critical comments by all the reviewers have helped us to improve the quality of our 
paper.

\section{References}

[1] M. Engels, "Wireless OFDM Systems," Kluwer Academic Publishers, New York, 2002.

[2] R. S. B. Ahmad and R. S. Burton, "Multi-Carrier_Digital_Communications: Theory and Applications of OFDM," Kluwer Academic Publishers, New York, 2002.

[3] M. S. Akram, "Pilot-based Channel Estimation in OFDM Systems," Master Dissertation, Technical University of Denmark, Denmark, 2007.

[4] S. Coleri, M. Ergen, A. Puri and A. Bahai, "Channel Estimation Techniques Based on Pilot Arrangement in OFDM Systems," IEEE Transactions on, Vol. 48, No. 3, September 2002, pp. 223-229.

[5] J. J. van de Beek, O. Edfors, M. Sandell, S. K. Wilson, W. Per and P. O. Börjesson, "On Channel Estimation in OFDM Systems," Vehicular Technology Conference, IEEE 45th, Vol. 2, 1995, pp. 815-819.

[6] H. Hijazi and L. Ros, "Rayleigh Time-varying Channel Complex Gains Estimation and ICI Cancellation in OFDM Systems," European Transactions on Telecommunications, Vol. 20, No. 1, 2009, pp. 782-796.

[7] R. Gonzalez and F. bader, "Time-Frequency Spacing Design for PACE in OFDM Systems," 14th IST Mobile and Wireless Communications Summit, Dresden, 2005.
[8] S. Ohno and G. B. Giannakis, "Capacity Maximizing MMSE-Optimal Pilots for Wireless OFDM over Frequency-Selective Block Rayleigh Fading Channels," IEEE Transactions on Information Theory, Vol. 50, No. 9, 2004, pp.2138-2145.

[9] P. Hoeher, S. Kaiser and P. Robertson, "Pilot-Symbol-Aided Channel Estimation in Time and Frequency," Kluwer Academic Publishers, USA, 1997.

[10] M. Sandell and O. Edfors, "A Comparative Study of Pilot-Based Channel Estimators for Wireless OFDM," Division of Signal Processing, Luleå University of Technology, Luleå, Sweden, September 1996.

[11] P. Y. Tsai and T. D. Chiueh, "Frequency-Domain Interpolation Based Channel Estimation in Pilot-Aided OFDM Systems," Vehicular Technology Conference, IEEE 59th, Vol. 1, 2004, pp. 420-424.

[12] L. Ros, "Réception multi-capteur pour un terminal radio-mobile dans un système d'accès multiple à répartition par codes," INP Grenoble, 2001.

[13] D. Tse and P. Viswanath, "Fundamentals of Wireless Communication," Cambrige University Press, UK, 2005.

[14] R. Negi and J. Cioffi, "Pilote Tone Selection for Channel Estimation in a Mobile OFDM System," Consumer Electronics. ICCE, Stanford, 1998, pp. 466-467.

[15] X. D. Cai and G. B. Giannakis "Error Probability Minimizing Pilots for OFDM with M-PSK Modulation over Rayleigh-Fading Channels," IEEE Transactions, Vol. 53, No. 1, 2004, pp. 145-160. 